\title{
Quantitative evaluation of thermodynamic parameters for thermal back-reaction after mechanically induced fluorescence change
}

\author{
Fuyuki Ito ${ }^{* a}$ and Takehiro Sagawa ${ }^{a}$ \\ Received (in $X X X, X X X)$ Xth $X X X X X X X X X 20 X X$, Accepted Xth $X X X X X X X X X 20 X X$ \\ DOI: 10.1039/b000000x
}

\begin{abstract}
Kinetics of the thermal back-reaction of $\beta$-diketonate boron difluoride complexes after mechanical perturbation were evaluated by fluorescence intensity changes for the first time, suggesting that the activation parameters of the reaction 10 intermediates governed intermolecular interactions such as hydrogen bonding assisted by substituent groups.
\end{abstract}

Organic fluorescent solids have been used widely in luminescent materials such as electroluminescent (EL) devices. ${ }^{1}$ Organic molecular solids or molecular assemblies are typically based on

15 van der Waals interactions such as $\pi-\pi$ interactions or hydrogen bonding interactions. These interactions promote the development of novel functions and properties, which differ from those in the monomer state. In the development of luminescent materials based on organic molecules, it is important to 20 understand the properties and functions, not only for the monomer species, but also for the assembled species. In photochromism in a molecular crystal system, for example, cooperative interaction results in a change of the crystal structure by the chromic reaction. Kobatake and Irie et al. report that 25 molecular crystals based on diarylethene chromophores with sizes ranging from 10 to $100 \mu \mathrm{m}$ exhibit rapid and reversible macroscopic changes in shape and size induced by UV and visible light. ${ }^{2}$ The densely packed crystals were proven to be useful to link molecular-scale events to the macroscale movement 30 of materials, which leads to mechanical perturbation. In contrast, it is believed that mechanical forces can perturb intermolecular interactions such as molecular distance. Over the past decade, mechano- and piezo-chromic luminescent effects on organic solids have been reported, ${ }^{3,4}$ especially on fluorescent materials. ${ }^{5-}$

${ }_{35}{ }^{7}$ The mechanochromic phenomena most probably originate from changes in intermolecular interactions induced by intermolecular distance changes.

The $\beta$-diketonate boron difluoride complex exhibits a fluorescence spectral change by mechanical perturbation 40 depending on its concentration. ${ }^{8-11}$ Fraser et al. reported a reversible mechanochromic fluorescent organic solid based on the 4-tert-butyl-4'-methoxydibenzoylmethane (trade name: avobenzone) boron difluoride complex, which shows different emission depending on the crystal type. ${ }^{12}$ The emission color 45 significantly red-shifts upon rubbing/smearing the samples. The samples then revert back to the original emission species over time. This is the first finding of a thermal-back type (t-type) fluorescent mechanochromism phenomenon. However, the kinetic and thermodynamic properties of t-type mechanochromic ${ }_{50}$ compounds have not been totally clarified. In this communication, we studied and quantitatively evaluated for the first time the thermodynamic parameters for thermal back-reaction after mechanically induced fluorescence change.

The molecular structure of boron difluoride $\beta$-diketonate ${ }_{55}$ complexes is shown in Chart 1. In this study we used 1-(4-tertbutylphenyl)-3-(4-methoxyphenyl)-1,3-propanedione (ab), 1,3bis-(4-methoxyphenyl)-1,3-propanedione (2a), and 1,3-bis-(4tert-butylphenyl)-1,3-propanedione (2b) as ligands. The boronation was performed by $\mathrm{BF}_{3} \cdot \mathrm{OEt}_{2}$ in $\mathrm{CH}_{2} \mathrm{Cl}_{2}$ according to a ${ }_{60}$ previous report. $^{12}$ The purification was carried out with column chromatography. A $2.0 \times 10^{-3} \mathrm{~mol} \cdot \mathrm{dm}^{-3}$ dichloromethane solution was dropped on paraffin-coated weighing paper, and then the solvent-evaporated sample was used for spectroscopic studies. Fluorescence spectra were recorded on a Shimadzu RF-5300PC ${ }_{65}$ fluorescence spectrophotometer. In order to apply a mechanical perturbation to the boron difluoride $\beta$-diketonate complexes, the samples were rubbed with a spatula. The temperature around the sample was controlled by a home-made system using a rubber heater (Hakko) and digital temperature controller (Omron E5CN$\left.{ }_{70} \mathrm{QT}\right)$.

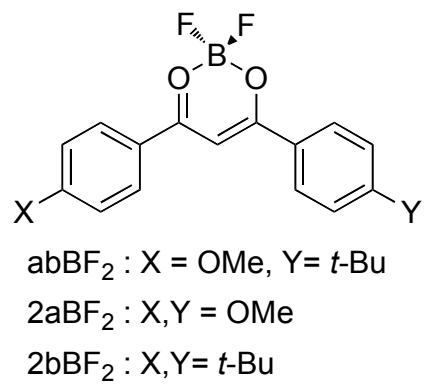

Chart 1 Molecular structure of boron difluoride $\beta$-diketonate complexes.

Fig. 1 shows fluorescence spectra of $\mathbf{a b B F}_{2}$ on paraffin-coated weighing paper at $303 \mathrm{~K}$. The fluorescence spectra were 75 normalized to the maximum intensity. The drop-casted $\mathbf{a b B F} \mathbf{b}_{\mathbf{2}}$ powder exhibited a blue emission that peaked at $460 \mathrm{~nm}$ as shown in blue in Fig. 1. The fluorescence spectrum originated from a single crystal or dendric solid as previously observed. ${ }^{12}$ After rubbing with a spatula, a new fluorescence band appeared 80 around $500 \mathrm{~nm}$ with a shoulder at $550 \mathrm{~nm}$, originating from the amorphous state of $\mathbf{a b B F}_{2}{ }^{12}$ The sample after rubbing emitted a yellow-green color under the UV lamp. The fluorescence 
intensity up to $530 \mathrm{~nm}$ decreased as time elapsed. After $1030 \mathrm{~min}$ the fluorescence spectrum showed peaks around 460 and $500 \mathrm{~nm}$, the emissive color appearing green under the UV lamp. These observations indicated that the yellow fluorescent species was 5 changed to green at room temperature, demonstrating $\mathbf{a b B F}_{2}$ as a t-type mechanochromic molecule.

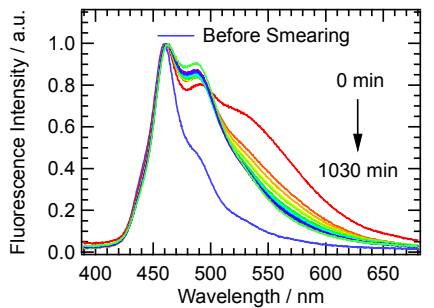

Fig. 1 Fluorescence spectra of $\mathbf{a b B F}_{2}$ at $303 \mathrm{~K}$. The excitation wavelength is $370 \mathrm{~nm}$.

10 In order to conduct a quantitative kinetic analysis for thermal back-reaction after mechanical perturbation, we measured the fluorescence intensity change. Fig. 2 shows changes in fluorescence intensity at $550 \mathrm{~nm}$ excited at $370 \mathrm{~nm}$ as a function of time. The temperature was maintained during the measurement 15 at (a) 296, (b) 303, and (c) $313 \mathrm{~K}$. The fluorescence intensity at $550 \mathrm{~nm}$ decreased with elapsed time, which can be reproduced with a double-exponential decay function obeying first-order kinetics. The rate constants of the faster $\left(k_{\mathrm{F}}\right)$ and slower $\left(k_{\mathrm{S}}\right)$ components obtained by least-squares fitting are listed in Table 1.

${ }_{20}$ The two rate constants increase with increasing temperature. The changes in fluorescence intensity quantitatively exhibit exponential decay from the manual smearing. No quantitative analysis on the ratio of the pre-exponential factor for the two decay components was performed.

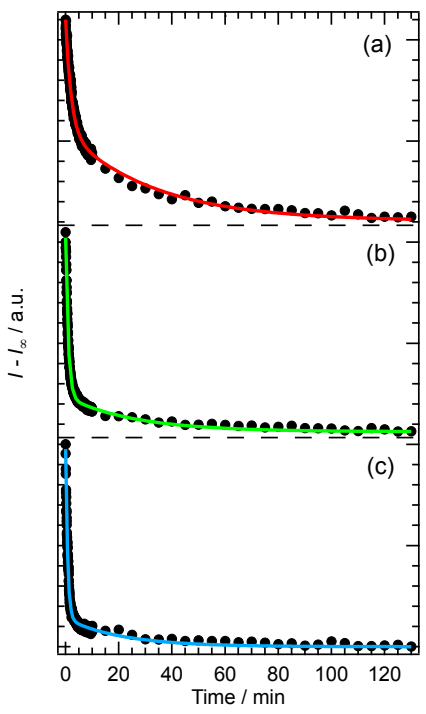

Fig. 2 Changes in fluorescence intensity of $\mathbf{a b B F}_{2}$ monitored at $550 \mathrm{~nm}$ as a function of time after mechanical perturbation. The temperature is (a) 296, (b) 303, and (c) $313 \mathrm{~K}$. The solid lines indicate the best-fitting curves based on a double-exponential decay function.
30 Table 1 Rate constant of thermal back-reaction for $\mathbf{a b B F} \mathbf{F}_{\mathbf{2}}$ from fitting of fluorescence decay.

\begin{tabular}{ccc}
\hline \multirow{2}{*}{ Temperature / K } & \multicolumn{2}{c}{ Rate constant $/ \mathrm{s}^{-1}$} \\
& $k_{\mathrm{F}}$ & $k_{\mathrm{S}}$ \\
\hline 296 & $8.06 \times 10^{-3}$ & $5.03 \times 10^{-4}$ \\
303 & $1.70 \times 10^{-2}$ & $6.43 \times 10^{-4}$ \\
313 & $2.25 \times 10^{-2}$ & $7.83 \times 10^{-4}$ \\
323 & $3.05 \times 10^{-2}$ & $9.72 \times 10^{-4}$ \\
333 & $8.52 \times 10^{-2}$ & $1.95 \times 10^{-3}$ \\
\hline
\end{tabular}

The fluorescence intensity at $550 \mathrm{~nm}$ excited at $370 \mathrm{~nm}$.

The $k_{\mathrm{F}}$ and $k_{\mathrm{S}}$ correspond to faster and slower rate constants.

The temperature dependence of the rate constants allows us to 35 determine the activation parameters of the amorphous-crystal phase transition of the boron difluoride $\beta$-diketonate complexes. The kinetic law of reaction $k=A \exp \left(-E_{\mathrm{a}} / R T\right)$ can be evaluated and used to provide the activation parameters, where $A, E_{\mathrm{a}}, R$, and $T$ are the pre-exponential factor, activation energy, gas constant, 40 and temperature, respectively. Fig. 3a shows the Arrhenius plot of the thermal back-reaction of $\mathbf{a} \mathbf{b} \mathbf{B} \mathbf{F}_{\mathbf{2}}$ together with a straight line by least-squares fitting. The $A$ and $E_{\text {a }}$ were estimated to be $1.05 \times 10^{6} \mathrm{~s}^{-1}$ and $45.8 \mathrm{~kJ} \cdot \mathrm{mol}^{-1}$ for $k_{\mathrm{F}}$, and $2.97 \times 10^{1} \mathrm{~s}^{-1}$ and 27.2 $\mathrm{kJ} \cdot \mathrm{mol}^{-1}$ for $k_{\mathrm{S}}$, respectively. The enthalpy and entropy of 45 activation energies $\left(\Delta H^{\ddagger}\right.$ and $\left.\Delta S^{\ddagger}\right)$ for the thermal back-reaction of $\mathbf{a b B F}_{2}$ were also estimated from the Eyring plot as shown in Fig. 3b, using the equation $k=\left(k_{\mathrm{B}} T / h\right) \exp \left(\Delta S^{\sharp} / R\right) \exp \left(-\Delta H^{\sharp} / R T\right)$, where $k_{\mathrm{B}}$ is the Boltzmann constant. The resulting $\Delta H^{\ddagger} \mathrm{s}$ are 43.2 $\mathrm{kJ} \cdot \mathrm{mol}^{-1}$ for the faster component and $24.6 \mathrm{~kJ} \cdot \mathrm{mol}^{-1}$ for the slower so component, and the $\Delta S^{\ddagger} \mathrm{s}$ are $-104 \mathrm{~J} \cdot \mathrm{K}^{-1}$ for the faster component and $-191 \mathrm{~J} \cdot \mathrm{K}^{-1}$ for the slower component. The estimated $\Delta S^{\ddagger}$ values are negative, suggesting the order parameter of the activated complex is higher than that of the initial amorphous phase. The thermodynamic findings confirmed that the thermal ${ }_{55}$ back-reaction of $\mathbf{a b B F}_{2}$ originates from the amorphous-crystal transformation. We think that the amorphous-crystal transformation correlates with the crystal growth process from the melt states. The $\Delta S^{*}$ for the faster component is lower than that for the slower component. The free energy barriers $\left(\Delta G^{*}\right)$ of ${ }_{60} \mathbf{a b B F}_{2}$ at $303 \mathrm{~K}$ for faster and slower components were 74.7 $\mathrm{kJ} \cdot \mathrm{mol}^{-1}$ and $82.5 \mathrm{~kJ} \cdot \mathrm{mol}^{-1}$, respectively. These findings indicate that the reaction via the lower-order activation complexes (transition state) is the dominant pathway for thermal backreaction after mechanical perturbation
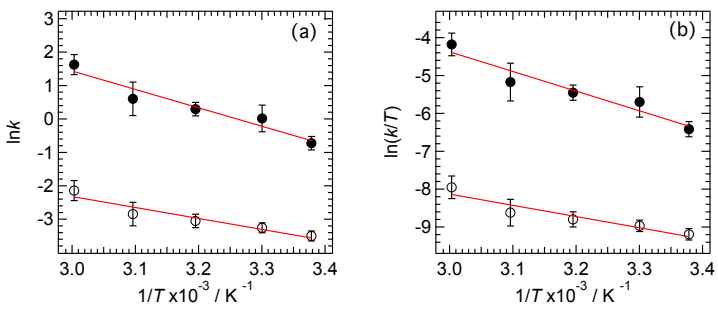

Fig. 3 Arrhenius (a) and Eyring (b) plots for thermal back-reaction of $\mathbf{a b B F}_{2}$. Closed and open circles correspond to faster and slower components.

In order to determine the kinetic and activation parameters for 70 the thermal back-reaction of $\mathbf{a b B F} \mathbf{b}_{2}$, we examined the substituent dependence for the reaction. Compound $\mathbf{a b B F _ { 2 }}$ has tert-butyl and 
methoxy substituents [the ligand is 1-(4-tert-butylphenyl)-3-(4methoxyphenyl)-1,3-propanedione]. Thus, we studied 1,3-bis-(4methoxyphenyl)-1,3-propanedione (2a) and 1,3-bis-(4-tertbutylphenyl)-1,3-propanedione (2b) as ligands next. Both $\mathbf{2} \mathbf{a} \mathbf{B} \mathbf{F}_{\mathbf{2}}$ 5 and $\mathbf{2} \mathbf{b B F} \mathbf{F}_{2}$ also showed a crystal-to-amorphous phase change subsequent to the mechanical perturbation, as confirmed by XRD spectra, and the substituent dependence of the rate constants and the activation parameters were determined in the same manner. These experimental data are included in the electronic 10 supplementary information (ESI). The activation parameters are listed in Table 2. The activation parameters of the faster and slower components of $\mathbf{a b B F _ { 2 }}$ are similar to that of the faster component of $\mathbf{2} \mathbf{a B F}_{\mathbf{2}}$ and the faster component of $\mathbf{2} \mathbf{b B F}$, respectively. This indicates that the kinetic modes of the faster 15 and slower components in $\mathbf{a b B F _ { 2 }}$ are related to the methoxy and tert-butyl substituents, respectively. The $\Delta G^{\ddagger}$ values at $303 \mathrm{~K}$ were estimated to be $77.2 \mathrm{~kJ} \cdot \mathrm{mol}^{-1}$ for the faster component and $83.3 \mathrm{~kJ} \cdot \mathrm{mol}^{-1}$ for the slower component for $\mathbf{2} \mathbf{a} \mathbf{B F}_{2}$, and 78.8 $\mathrm{kJ} \cdot \mathrm{mol}^{-1}$ for the faster component and $84.3 \mathrm{~kJ} \cdot \mathrm{mol}^{-1}$ for the slower 20 component for $\mathbf{2 b B F}_{2}$. It can be seen from comparing these values with $\mathbf{a b B F} 2$ that $\Delta G^{\ddagger}$ is not dependent on the substituent group. These findings indicate that the substituent group of the boron difluoride $\beta$-diketonate complexes does not affect the energy barrier for the system, but does affect the thermal back25 reaction rates. The formation process of the activated complex (transition state) is governed by the entropic term. The $\Delta H^{*}$ value is much larger than the formation energy by the van der Waals interaction (generally $1 \mathrm{~kJ} \cdot \mathrm{mol}^{-1}$ ), but is comparable to the dipolar interaction and hydrogen bonding interaction $\left(10-150 \mathrm{~kJ} \cdot \mathrm{mol}^{-1}\right)$.

30 Fraser et al. suggested that the multiple interactions, such as strong dipolar nature of the molecules, arene stacking and $\mathrm{C}$ (arene) $-\mathrm{H} \cdots \mathrm{F}$ hydrogen bonds, may be the driving force for the aggregation of the boron difluoride $\beta$-diketonate complexes upon thermal treatment or recovery after smearing. ${ }^{10-13}$ Because the

35 kinetic mode related to the methoxy substituent preferentially promotes crystal reformation over the tert-butyl substituent, the driving force and transition state for the crystallization of $\mathbf{a b B F}_{\mathbf{2}}$ will contribute not only to the $\mathrm{C}$ (arene) $-\mathrm{H} \cdots \mathrm{F}$ interaction, but also to the $\mathrm{C}$ (arene) $-\mathrm{H} \cdots \mathrm{O}$ (methoxy) interaction. ${ }^{14}$ Preliminary 40 studies of the IR spectra of $\mathbf{a b B F}_{2}$ indicate that the bands attributed to $\mathrm{C}-\mathrm{H}$ stretching $\left(850 \mathrm{~cm}^{-1}\right)$ and out-of-plane bending modes (1560-1580 $\left.\mathrm{cm}^{-1}\right)$ were dramatically changed before and after the smearing process (see ESI). Thus, it is found that intermolecular interactions, especially the hydrogen bonding 45 interaction, influence the mechanofluorochromism for the boron difluoride $\beta$-diketonate complexes in terms of kinetic and thermodynamic parameters.

Table 2 Kinetic and activation parameters of the thermal back-reaction.

\begin{tabular}{|c|c|c|c|c|c|}
\hline Compound & Component & $\begin{array}{c}E_{\mathrm{a}} \\
\mathrm{kJ} \cdot \mathrm{mol}^{-1}\end{array}$ & $\begin{array}{l}A \\
\mathrm{~s}^{-1}\end{array}$ & $\begin{array}{c}\Delta H^{*} \\
\mathrm{~kJ} \cdot \mathrm{mol}^{-1}\end{array}$ & $\begin{array}{c}\Delta S^{+} \\
\mathrm{J} \cdot \mathrm{K}^{-1}\end{array}$ \\
\hline \multirow{2}{*}{$\mathbf{a b B F}_{2}$} & $k_{\mathrm{F}}$ & 45.8 & $1.05 \times 10^{6}$ & 43.2 & -104 \\
\hline & $k_{\mathrm{S}}$ & 27.2 & $2.97 \times 10^{1}$ & 24.6 & -191 \\
\hline \multirow{2}{*}{$2 \mathbf{a B F}_{2}$} & $k_{\mathrm{F}}$ & 44.1 & $2.36 \times 10^{5}$ & 41.6 & -116 \\
\hline & $k_{\mathrm{S}}$ & 33.6 & $2.82 \times 10^{2}$ & 31.1 & -172 \\
\hline \multirow{2}{*}{$2 \mathrm{bBF}_{2}$} & $k_{\mathrm{F}}$ & 23.1 & $2.65 \times 10^{1}$ & 20.5 & -192 \\
\hline & $k_{\mathrm{S}}$ & 17.1 & $2.70 \times 10^{-1}$ & 14.5 & -230 \\
\hline
\end{tabular}

In summary, we have studied and evaluated quantitatively the thermodynamic parameters for the thermal back-reaction of $\mathbf{a b B F}_{2}$ after a mechanically induced fluorescence change. The changes in fluorescence intensity exhibited exponential decay after the mechanical perturbation that depended on temperature. 5 The activation parameters of the amorphous-crystal phase transition suggested that the transition state to the activated complex was higher than that of the initial amorphous phase based on the negative $\Delta S^{\ddagger}$ values. The substituent groups influenced the thermal back-reaction rates, which most probably

${ }_{60}$ controlled intermolecular interactions such as hydrogen bonding between the boron difluoride $\beta$-diketonate complexes. These findings will guide the design of mechanofluorochromic reactions by controlling the substituent groups, and also influence studies on the kinetics and thermodynamics of the crystal nuclei ${ }_{65}$ formation process from a melted state to a crystal state. Detailed studies on the substitution dependence of the thermal backreaction by thermal analysis (DSC) of the crystals and IR spectral changes are now in progress.

The authors thank Dr. Kennosuke Itoh (Shinshu University) 70 for the NMR measurements. This work was partly supported by Nanotechnology Platform Program (Molecule and Material Synthesis) of the MEXT, Japan.

\section{Notes and references}

a Department of Chemistry, Faculty of Education, Shinshu University, 675 ro, Nishinagano, Nagano 380-8544, Japan. Fax: +81-26-238-4114; Tel: +81-26-238-4114; E-mail: fito@shinshu-u.ac.jp

$\dagger$ Electronic Supplementary Information (ESI) available: [XRD spectra, fluorescence spectra and Arrhenius and Eyring plots of $\mathbf{2} \mathbf{a B F} \mathbf{2}$ and $\mathbf{2} \mathbf{b B} \mathbf{B F}_{\mathbf{2}}$, IR spectra of $\mathbf{a b B F}_{2}$ ]. See DOI: 10.1039/b000000x/

801 M. Schwoerer and H. C. Wolf, Organic Molecular Solids, Wiley-VCH, Weinheim, Germany, 2007.

2 S. Kobatake, S. Takami, H. Muto, T. Ishikawa and M. Irie, Nature, 2007, 446, 778.

3 Y. Sagara and T. Kato, Nat. Chem., 2009, 1, 605.

${ }_{85} 4$ Z. G. Chi, X. Q. Zhang, B. J. Xu, X. Zhou, C. P. Ma, Y. Zhang, S. W. Liu and J. R. Xu, Chem. Soc. Rev., 2012, 41, 3878.

5 Y. Sagara, T. Mutai, I. Yoshikawa and K. Araki, J. Am. Chem. Soc., 2007, 129, 1520.

6 H. Ito, T. Saito, N. Oshima, N. Kitamura, S. Ishizaka, Y. Hinatsu, M.

90 Wakeshima, M. Kato, K. Tsuge and M. Sawamura, J. Am. Chem. Soc., 2008, 130, 10044.

7 Y. Ooyama, G. Ito, H. Fukuoka, T. Nagano, Y. Kagawa, I. Imae, K. Komaguchi and Y. Harima, Tetrahedron, 2010, 66, 7268.

8 A. G. Mirochnik, E. V. Fedorenko, T. A. Kaidalova, E. B. Merkulov,

95 V. G. Kulyavyi, K. N. Galkin and V. E. Karasev, J. Lumin., 2008, 128, 1799.

9 A. Sakai, M. Tanaka, E. Ohta, Y. Yoshimoto, K. Mizuno and H. Ikeda, Tetrahedron Lett., 2012, 53, 4138 .

10G. Q. Zhang, J. P. Singer, S. E. Kooi, R. E. Evans, E. L. Thomas and C. L. Fraser, J. Mater. Chem., 2011, 21, 8295.

11T. Liu, A. D. Chien, J. Lu, G. Zhang and C. L. Fraser, J. Mater. Chem., 2011, 21, 8401 .

12 G. Q. Zhang, J. W. Lu, M. Sabat and C. L. Fraser, J. Am. Chem. Soc., 2010, 132, 2160.

10513 X. Zhang, C.-J. Yan, G.-B. Pan, R.-Q. Zhang and L.-J. Wan, J. Phys. Chem. C, 2007, 111, 13851.

14 R. Davis, N. S. S. Kumar, S. Abraham, C. H. Suresh, N. P. Rath, N. 
Tamaoki and S. Das, J. Phys. Chem. C, 2008, 112, 2137. 